\title{
Routine operation of an Elliott 903 computer in a clinical chemistry laboratory
}

\author{
L. G. WHITBY AND D. SIMPSON \\ From the Department of Clinical Chemistry, Royal Infirmary of Edinburgh
}

SYNOPSIS Experience gained in the last four years concerning the capabilities and limitations of an 8K Elliott 903 (18-bit word) computer with magnetic tape backing store in the routine operation of a clinical chemistry laboratory is described. Designed as a total system, routine operation has latterly had to be confined to data acquisition and process control functions, due primarily to limitations imposed by the choice of hardware early in the project. In this final report of a partially successful experiment the opportunity is taken to review mistakes made, especially at the start of the project, to warn potential computer users of pitfalls to be avoided.

The Report of the Working Party on Computers in Medicine (British Medical Association, 1969) listed the benefits obtainable under four main headings: (1) saving of direct costs of existing operations; (2) increased quality of work mainly through eliminating errors but also in terms of legibility, comprehensiveness, etc; (3) saving of time and of work which is not readily translated into cash equivalents; (4) production of summaries, reports, and evaluations of work done at a time when they are still topical.

The fundamental objective of any health service computer system should be the provision of better patient care. A clinical chemistry computer system can improve patient care by helping to provide a quicker, more efficient service of more reliable information required for the diagnosis and treatment of disease.

In highly mechanized laboratories, where manual methods of data acquisition and processing are in use, trained technical staff spend up to $30 \%$ of their time on routine clerical work, including the preparation of day sheets and work sheets, acquisition of data from analytical equipment, calculation and correlation of results, transcription of information to report forms, and maintenance of laboratory and patient records (Rappoport, 1965; Robinson, 1971). The employment of skilled staff for such work is wasteful, and its repetitive nature is conducive to errors.

By introducing a computer into the laboratory, it is possible to improve the administrative activities which depend upon accurate information carried on

Received for publication 8 May 1973. test request forms. It is also possible to reduce errors due to inaccurate reading of recorder charts, to perform calculations automatically; to eliminate manual transcription, to improve quality control procedures, to provide efficient storage and recall of results, and to improve the presentation and interpretation of reports.

Further benefits may be obtained from the analysis of computer-held cumulative files of laboratory work. The considerable amount of data capable of being accessed by a computer should be more readily examined than data in patients' notes, and could be analysed to find ways of facilitating the early detection of disease.

In the last 10 years much experience has been gained in the United Kingdom with computers in clinical chemistry laboratories, and most of the foregoing advantages that derive from computerdependent operations have been achieved by one or other system, although no system has succeeded in all these respects. The American laboratory computer scene has recently been reviewed (Johnson, 1971), and it would seem that there too, despite much effort, there is as yet no computer system able to be widely recommended for installation into the clinical laboratory. The time seems appropriate to review our experience, with a computer dedicated to the work of a clinical chemistry laboratory, and to summarize the lessons to be learned from this partially successful experiment.

\section{Summary of the Elliott 903 Project}

In 1966 this laboratory embarked on a programme of research and development, in collaboration with 
Elliott Medical Automation Ltd, ${ }^{1}$ and with the backing of the National Research Development Corporation (NRDC). The aims were to achieve, with a dedicated computer system based on an $8 \mathrm{~K}$ (18-bit word) Elliott 903 computer, the improvements in laboratory service referred to in the introduction. The terms of the agreement required Elliotts to be responsible for writing the complete software for the system. Apart from assisting in the initial systems design, the main tasks of this department's staff early in the venture were to provide details on requesting procedures, the work flow of the laboratory, the operation of mark I AutoAnalyzers, ${ }^{2}$ and the information to be included on report forms. At a later stage, the laboratory carried out the testing, validating, or rejecting of individual programs as they became available, and repeated these tests for each revised or integrated set of programs.

\section{The System in Routine Operation}

The hardware configuration and the features of special hardware developed by Elliotts for the laboratory have been described (Whitby and Simpson, 1969; Griffiths and Carter, 1969; Simpson, Sims, Harrison, and Whitby, 1971). These earlier accounts also described the system's software and operational procedures, with an indication of the capabilities and limitations of the system in routine operation. This section will deal mainly with points that have emerged since the earlier accounts were published. At the outset of the project, the laboratory's work load for specimens from patients was approximately 400000 determinations a year; this figure has risen to 770000 determinations a year, and is made up almost entirely of discretionary (Whitehead, 1972) requests.

1 INPUT OF PATIENT IDENTIFICATION AND TEST REQUEST DATA, GENERATION OF WORK SHEETS, AND STATUS REPORTS

Patient identification in the Royal Infirmary is based on the date of birth; no unique patient registration number is issued. As a result, for unique identification, it is necessary to use additional information such as surname, initials, sex, etc. Because of the variability of this information and the difficulty of using computer check routines, a conversational mode of input was initially selected. This had to be abandoned because of the slowness of tape searches, particularly when the computer was being used simultaneously to monitor AutoAnalyzers. It was replaced by an off-line procedure.

${ }^{1}$ Elliott Medical Automation Ltd, Elstree Way, Borehamwood, Herts. 'Technicon Instruments Co, Basingstoke, Hants.
Typing errors detected by manual checking of the off-line input, but not detected by the computer, revealed that the total error rate was $0.5 \%$. This includes transposition of digits in the date of birth and other transcription errors, but does not include errors in the information provided on hand-written request forms; these would not be detectable by the computer under any circumstances, but could more than double the total error rate. The error rate for the alpha characters in the surname was more than twice that for numerical characters.

Although the computer has been programmed for the generation of work sheets and status reports listing unallocated work, it has proved impossible to use it routinely for these purposes because even the off-line data entry procedure has been too slow to keep up with the arrival of request forms and specimens-in this laboratory, up to 1000 request forms daily at present. The advantages of punched card requesting procedures are discussed later.

2 DATA ACQUISITION AND PEAK DETECTION FROM AUTOANALYZERS, STANDARD CURVE VALIDATION

The principal features of the peak detection routines for use with standard colorimeters and the mark II flame photometer, when operated with AutoAnalyzer I equipment, have been described (Whitby and Simpson, 1969). Only minor adjustments to parameters in the programs, undertaken in the laboratory, have since been needed to acquire signals from the fluorimeter unit and the mark IV flame photometer, operated with AutoAnalyzer I equipment, and to accept the output from single channel AutoAnalyzer II equipment. The computer has not been used to monitor analytical equipment other than AutoAnalyzers I and II. Attempts to improve the peak detection procedures so as to reject all peaks that are faulty due to inadequate sampling have met with some success but the modified procedures considerably increased the complexity of the programs. Using unmodified programs, failure to reject 'short samples' in routine operation means that $0 \cdot 1-0.2 \%$ of faulty peaks are being accepted. However, the magnitude of the error of the incorrectly accepted samples does not exceed $12 \%$ of the 'true' concentrations of materials being analysed in the affected samples; grosser degrees of 'short sampling' result in rejection of the specimen by the standard program.

Variation in the shape of calibration curves has meant that there are limitations to the usefulness of computer validation of curves based on the system of linear interpolation (Whitby and Simpson, 1969), and the checking of calibration curves by visual inspection is still an important part of curve 
validation. The use of polynomial expressions for calibration and validation of standard curves was investigated, but the magnitude of variation from day to day in coefficients proved to be such that it would have been necessary to set very wide limits for coefficients to prevent rejection of curves which, on visual examination, appeared acceptable.

The Compromise between Individual Capability and Performance as a Total System

Whitby and Simpson (1969) described the many individual operations satisfactorily programmed for the Elliott 903 computer, and Simpson et al (1971) reported timings for various operations when the computer was used to perform these activities in an integrated manner. Attention was drawn to serious inadequacies of the system, at least for an annual work load over 500000 determinations. Minor improvements have since been made in some of the timings by program changes, but these have not begun to make the system function as a whole within an acceptable time-scale. Instead, only some of the software capabilities are now being utilized routinely, but on a seven day/week basis in the last three years, principally on-line operations, as follows: (1) acquisition of raw data from as many as 19 different determinations on up to 12 AutoAnalyzer channels at one time; (2) peak detection and validation, and standard curve acceptance; (3) calculation of results after correction for instrumental drift; (4) output of results identified by cup number; (5) calculation of batch means and standard deviations for analyses of specimens from patients.

The system has proved unable to perform, within the normal working day, several functions originally specified for the total system. The features now omitted include: (A) input to the computer of patient and test data for all requests received; (B) preparation of work sheets for automated and manual tests, and status reports; (C) immediate recognition of faulty results for quality control sera; (D) compilation of cumulative records on magnetic tape; (E) preparation of cumulative reports, end-of-day summaries, etc.

In summary, the laboratory has a hardware configuration programmed to operate as a total system. Because some operations are performed too slowly, under routine conditions, the total system approach has been abandoned and the equipment is instead being used as little more than a data acquisition system. This does still offer many benefits, however, since manual reading of AutoAnalyzer charts is avoided thereby eliminating reading errors. However, transcription of results from the computer printout to manually prepared work sheets, and further transcription of results from work sheets to patient reports are required, and the present method of use thus fails to reduce, let alone eliminate, transcription errors that can occur at both stages.

\section{Cost-benefit Considerations}

An important part of the evaluation of any computer system is a cost-benefit assessment (Review for the National Health Service, 1971). The benefits derived by this laboratory from the routine operation of its computer are: (A) throughput of work has continued to rise exponentially with only a small increase in staff, meaning an increase in the 'productivity' of staff and decrease in average cost per determination; (B) reduction in errors, by eliminating reading of recorder charts and subsequent calculation of results by technicians; (C) quality control statistics calculated in time to influence laboratory decisions.

Undoubtedly these are benefits, but they represent only a partical contribution when compared with the aims of the total system. They represent the service return on a capital investment of about $£ 32000$, at least an equal outlay by the manufacturer on software development, plus a considerable investment (not closely costed) of effort by laboratory personnel.

When the system's limitations in routine operation had been defined, it was at first thought that these might be overcome by enhancing it with a lineprinter and edge-punched card requesting facilities (Simpson et al, 1971). Later assessments led to the conclusion that it would be inappropriate to extend the present system because of (1) inability to interface an Elliott 903 computer to a fast access backing store such as a magnetic disc; (2) knowledge that a similar 8K Elliott 903 system, but provided additionally with a line-printer and having input of test request data facilitated by the use of edge-punched cards, could cope on a service basis with an annual workload of about 300000 determinations only when operated on a very tight schedule and on a double shift (Flynn, F. V., and Piper, K. A., personal communication); (3) the decision by Elliotts to advocate the Elliott 905 computer rather than the 903 for use in a clinical chemistry laboratory.

Lessons for the Future: a Consideration of Mistakes Made

The outcome of this programme of experiments, which lasted nearly seven years, can at best be rated a qualified success. However, some mistakes can be defined and their definition could be helpful to laboratories in determining their approach to computer usage, and especially to those who might 
believe that the introduction of computer-assisted operations is as easy as some computer salesmen still make out. Clinical chemistry continues in the stage of 'computer experiment', as reviews (eg, Johnson, 1971) show that there is no system currently operating which offers all the benefits listed in the opening paragraphs of this paper.

\section{A CHOICE OF HARDWARE}

Decisions about the hardware configuration (Whitby and Simpson, 1969) were taken too early before a detailed analysis had been made of the requirements to be met by the system. Furthermore, the initial outlay on hardware was arbitrarily limited to $£ 30000$, this ceiling figure having been determined by theoretical economic arguments rather than practical scientific assessment. Systems analysis and functional specification for computer-dependent operations should be worked out in considerable detail before selecting the computer manufacturer and the most appropriate hardware configuration for performing these operations. Cost-benefit considerations should also influence the level of investment, but tight constraints should not be introduced too early in the design stage.

\section{B SOFTWARE PROVISION}

Our worst mistake, not recognized at the time as even a possible error, was acquiescence in the arrangement whereby laboratory-based programming staff were not employed for writing the software. Difficulties in adequately acquainting the systems analysts and computer programmers with essential details relating to the laboratory's operations, when the software team was based 400 miles away and only made brief visits to the laboratory, undoubtedly contributed to the delays in program writing and subsequent debugging, and probably to the shortcomings of the system as finally developed.

Other difficulties arose over commercial considerations of confidentiality relating to the anticipated value of software written by the manufacturers. Although laboratory staff have become familiar, over the years, with much of the detailed content of these programs, delays in providing adequate documentation and periodical changes in the manufacturer's programming team meant that laboratory staff were never adequately informed and were, therefore, limited in their ability to contribute usefully to improving the software in the light of operational experience. Only recently, nearly five years after the delivery of the computer and following the manufacturers' decision to disband their software team, has detailed documentation of the programs been provided. This documentation is incomplete in several important respects, has been supplied too late to be of much use, and an extra charge was levied for these incomplete documents.

The fundamental importance of having software teams on site is now widely recognized, except perhaps for genuine turnkey operations, but even with these laboratory staff must be properly informed about the software and have adequate documentation from the time computer usage begins. Difficulties in programming laboratory operations should not be underestimated, and it is probably false economy to add to these by arbitrary hardware constraints, especially now that core store costs are so much less than a few years ago. For the next generation of laboratory computers it would seem that $16 \mathrm{~K}$ of core is the minimum required for a clinical chemistry system supporting a laboratory with a workload of 500000 to 1000000 tests a year, but $32 \mathrm{~K}$ would ease the programming tasks considerably.

C THE TOTAL SYSTEMS APPROACH AND SOFTWARE UPDATING

When the system was first proposed, the suitability of the Elliott 903 computer for laboratory computing was unknown, and the limitations of an $8 \mathrm{~K}$ configuration, with only slow access to a backingstore that was restricted to magnetic tape units, were not appreciated. There were major problems in writing the initial software. Furthermore, because a total systems approach had been adopted, there were difficulties and considerable expense whenever even a minor change in programming was required, either as a result of experience in operation or developments in analytical practice. Updating software can be a very expensive aspect of continued and developing computer usage, but few laboratories would want to freeze their methods of analytical operation just because a computer has been installed. Other computer users have commented on the expense of 'zapping', or paying for program updates provided by the suppliers of total computer systems (Johnson, 1971). Dependence on outside sources for such updating support does not free the laboratory staff from the responsibility of checking each new set of programs to ensure that they perform satisfactorily what they purport to do.

If a laboratory recruits its own computer programmers, it should be in a much better position to continue its own software development. If, in addition, the applications programming can be undertaken in a machine-independent high-level language, this should make easier the interchange between different users of tested and clearly documented programs. With the Elliott 903, its high level language (SIR) is virtually machine-dependent, and the software would have needed to be entirely 
rewritten except perhaps for use with other 900 series computers.

D THE HOSPITAL INTERFACE: THE IMPORTANCE OF DATA PROCESSING

A clinical chemistry laboratory, in providing a diagnostic service, receives specimens accompanied by request forms and, in due course, issues reports bearing the results of the analytical work. The need to interface the laboratory efficiently with the requirements of its users places emphasis on the importance of requesting and reporting procedures. Whitehead, Becker, and Peters (1968) showed, for instance, how a simple but effective system of requesting procedures and data processing could be introduced to facilitate laboratory work, fitting in smoothly with the hospital's operations.

In this laboratory's programme of work, attention initially focused on the acquisition of signals from AutoAnalyzers, to provide computer-assisted operations that would replace and improve an earlier off-line system (Flynn, 1965, 1966; Flynn, Piper, and Roberts, 1966; Whitby, Proffitt, and McMaster, 1968), and insufficient thought was given to the hospital interface. The interest and cooperation of the Medical Records Department should have been sought and obtained at a very early stage. The difficulty of building up files of laboratory data relating to individual patients would have been greatly reduced by having punched card requesting procedures, preferably associated with the adoption of a unique numbering system for identification purposes.

On the output side, the goal of computer-prepared cumulative reports has proved unattainable. Unfortunately, in the belief that cumulative reports would be provided by the computer, no thought was given to less ambitious formats for computer-prepared reports such as the wage-slip variety described by Whitehead et al (1968). Considerations of difficulty, and so expense, ruled out the possibility of enhancing the system and rewriting the software so that the output programs could be run while the computer is still monitoring AutoAnalyzers, and timing limitations prevent the operation of the existing system in two separate modes, (1) for AutoAnalyzer monitoring and (2) later for report preparation.

In any fresh approach to laboratory computing, we would probably place less emphasis on the importance of monitoring laboratory equipment by computer, and more on the requirements for an efficient data-processing system. Besides defining the output format that reports should normally have, much more consideration would be given to fail-soft systems of reporting with a view to minimizing the need to fall back on manual procedures.
E GOING LIVE TOO EARLY: THE IMPORTANCE OF STAFF EDUCATION

Early experience with the system (Whitby and Simpson, 1969) demonstrated many of its capabilities. These assessments involved much effort as they required extensive parallel operation. Although the laboratory staff (in this context, more particularly the technical staff) had been kept fully in touch with the programme of experiments, there was understandable impatience to derive some clear-cut benefits from the computer. As a result, the computer began to be used routinely once the AutoAnalyzer monitoring and simple calculation routines had been fully proved, but before problems relating to request form entry, work scheduling, records storage and report generation had been fully explored, let alone solved. Using the computer routinely as a data acquisition system immediately placed constraints upon the opportunities for experimental work on the data processing functions.

Parallel operation is demanding on staff responsible for providing a routine service, and it is asking a lot of technicians for them to forego any obvious reward for their efforts while prolonged experiments that require their cooperation continue, such as the seemingly needless repetition of previously completed work that is often inevitable when a new set of programs has to be tested. Nevertheless, for eventual success, parallel operation must be persisted with until the main programme of experiments relating to service usage is complete. This demands careful attention to informing staff about objectives, educating the department as a whole about the plan of work, and keeping staff regularly in touch with developments, whether these be successes or failures. We probably did not pay sufficient heed to these requirements.

\section{Discussion}

The difficulties encountered in attempting to provide a data acquisition and data processing system for a routine clinical chemistry laboratory with a small dedicated computer have been described and some have already been discussed. The principal reasons for failing to achieve this objective can be summarized under three general headings: (1) inadequate hardware for the job on hand, (2) lack of flexibility of software and problems associated with the modification and updating of programs, and (3) specific problems associated with the clinical chemistry laboratory in the Royal Infirmary, Edinburgh.

The basic hardware required for the system was seriously underestimated. In addition, the non-modular nature of the system's software and the 
consequent difficulties in modifying and updating programs proved a major and continuing source of delays. Programs should be sufficiently flexible to permit changes in basic characteristics to be effected in a simple manner. The provision of clear and concise documentation is of vital importance in any development system.

Most of the difficulties associated with the input of patient and test data stemmed from the fact that no unique hospital registration number is available in the Royal Infirmary, Edinburgh. The use of the non-unique date of birth number made the input of data slow, cumbersome, and imprecise. Conversational mode is a fairly efficient method of linking existing patient records with current test requests but is very wasteful of computer time. Off-line data preparation procedures make record linkage more difficult and give rise to unnecessary duplication of records for individual patients, but are less demanding of computer time.

The laboratory has now adjusted to the limitations of the Elliott 903 system, and is using it routinely for data acquisition from AutoAnalyzers. This is perhaps a fortunate compromise as the range of automatic equipment available to clinical chemists is widening rapidly, although much of it still has to pass user-evaluation tests. It is not yet known which new equipment will prove suitable for routine service operation, but laboratory computing plans will need to take account of the increasing tendency for each complex analytical system (eg, AGA AutoChemist, ${ }^{1}$ Vickers MC $300^{2}$, Technicon SMAC) to include its own process-control computer. These instrumental factors could well have a marked influence on the further development of dedicated laboratory computer systems, and can certainly be expected to affect the total hardware requirement. The various laboratory configurations are also likely to be influenced by the availability of access to dataprocessing installations, either in major hospitals or at regional hospital board offices, and by developments in communications technology.

Opportunities for computer assistance in the many and various activities of a clinical chemistry laboratory are still open to experiment as no single satisfactory solution to these laboratories' needs has been identified. For the potential user, apart from defining his needs, the most important lesson to be derived from the review by Johnson (1971) is the advisability of going to see computers at work rather than passively accepting manufacturers' descriptions of capabilities. Unfortunately, considerations of cost seem already to be directing attention to the possibilities of forcing standardization (Review for the National Health Service, 1971).

This work has been generously supported by the South-Eastern Regional Hospital Board, Scotland. We would also like to thank the many members of departmental staff who have been involved in the project.

\section{References}

British Medical Association (1969). Computers in Medicine: Report of the Working Party (B.M.A. Planning Unit Report No. 3), p. 41. British Medical Association, London.

Flynn, F. V. (1965). Computer-assisted processing of biochemical test data. In Progress in Medical Computing, pp. 46-51. Elliott Medical Automation, London.

Flynn, F. V. (1966). Use of a computer by a clinical chemistry service. Proc. roy. Soc. Med., 59, 779-782.

Flynn, F. V., Piper, K. A., and Roberts, P. K. (1966). Equipment for linking the AutoAnalyzer to an off-line computer. J. clin. Path., 19, 633-639.

Griffiths, P. D., and Carter, N. W. (1969). On-line acquisition of the output of AutoAnalyzers. J. clin. Path., 22, 609-616.

Johnson, L. (1971). Clinical Laboratory Computer Systems: A Comprehensive Evaluation. Lloyd Johnson Associates, Northbrook, Illinois.

Rappoport, A. E. (1965). Introduction to a computer-assisted clinical laboratory. In Laboratary Management: Symposium on Computer-assisted Pathology, Miami, 1964. College of American Pathologists, Chicago.

Review for the National Health Service (1971). Using Computers to Improve Health Services. Department of Health and Social Security, London.

Robinson, R. (1971). Clinical Chemistry and Automation: A Study in Laboratory Proficiency. Griffin, London.

Simpson, D., Sims, G. E., Harrison, M. I., and Whitby, L. G. (1971). Equipment for linking the AutoAnalyzer on-line to a computer. J. clin. Path., 24, 170-176.

Whitby, L. G., Proffitt, J., and McMaster, R. S. (1968). Experience with off-line processing by computer of chemical laboratory data. Scot. med. J., 13, 181-191.

Whitby, L. G., and Simpson, D. (1969). Experience with on-line computing in clinical chemistry. J. clin. Path., 22, Suppl. (Coll. Path.), 3, 107-124.

Whitehead, T. P. (1972). Multiple analyses and their use in patient investigation. In The Pathological Basis of Medicine, edited by R. C. Curran and D. G. Harnden, pp. 572-581. Heinemann, London.

Whitehead, T. P., Becker, J. F., and Peters, M. (1968). Data processing in a clinical biochemistry laboratory. In Computers in the Service of Medicine, edited by G. McLachlan and R. A. Shegog. Vol. 1, pp. 113-133. Oxford University Press, London. 